\title{
LOW-COST, COMPACT UWB ANTENNA WITH FREQUENCY BAND-NOTCH FUNCTION
}

\author{
H. J. Visser* \\ * Holst Centre - TNO \\ P.O. Box 8550 \\ $5605 \mathrm{KN}$ Eindhoven, The Netherlands \\ E-mail: huib.j.visser@tno.nl
}

Keywords: Antennas, Bandstop filters, Design methodology, Dipole Antennas, Microstrip.

\begin{abstract}
Low-cost, compact, circular planar dipole UWB antennas have been designed, based on the 'two-penny dipole'. One of the dipole arms has been integrated with the ground plane of a microstrip transmission line, the strip being connected to the other dipole arm. The use of microstrip in stead of stripline technology hardly degrades the radiation characteristics, but simplifies the antenna architecture considerably.

Up to $6 \mathrm{GHz}$ the azimuthal gain variation does not exceed $3 d B$. For frequencies in excess of $6 \mathrm{GHz}$ the antenna becomes too long, resulting in the forming of elevational lobes and large azimuthal gain variations. Shortening the antenna, results in gain-uniformity in azimuth for high frequencies at the cost of a resonance shift beyond $3 \mathrm{GHz}$. As shown by the first full-wave simulations, this shortening may be realised without compromising the in-band return loss specifications. First measurements performed with antennas realised on FR4 - although hindered by cable-current effects - indicate the correctness of this approach.

By inserting a slot in the upper arm of the dipole or inserting a spurline filter in the microstrip part of the antenna, a stop band from $5 \mathrm{GHz}$ to $6 \mathrm{GHz}$ may be realised.
\end{abstract}

\section{Introduction}

High-data-rate wireless communications need wide bandwidths. In the Ultra-Wideband (UWB) frequency band from $3.1 \mathrm{GHz}$ to $10.6 \mathrm{GHz}$, information may be spread over a large bandwidth at low power levels thus creating the possibility to share the spectrum with other users. To prevent interference with existing wireless systems, like IEEE 802.11a WLAN, stop band characteristics are required from $5 \mathrm{GHz}$ to $6 \mathrm{GHz}$. In general, the UWB system and thus the antenna should be small and inexpensive. These constraints, added to the low power levels, make the antenna a critical component.

\subsection{Ultra Wide Band antennas}

From the 1930's on, antenna engineers have been searching for wideband antenna elements. Soon they discovered that, starting from a dipole or monopole antenna, thickening the arms resulted in an increased bandwidth. The reason for this is that for a thick dipole or monopole antenna, the current distribution is - unlike for the thin dipole and monopole - not longer sinusoidal. While this hardly affects the radiation pattern of the antenna, it severely influences the input impedance, [1]. This band-widening effect is even more severe if the thick dipole is given the shape of a biconical antenna. A further evolution may be found in dipole and monopole antennas formed by spheres or ellipsoids, [2]. Figure 1 shows the evolution from thin-wire dipole antenna towards spherical dipole antenna.

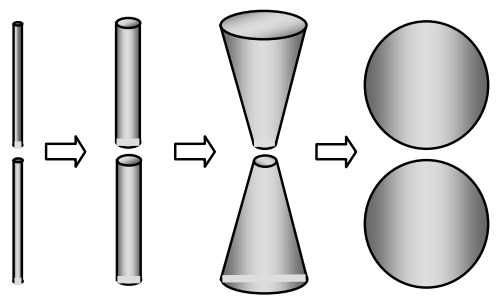

Figure 1: Evolution from small-band thin-wire dipole antenna towards broad-band spherical dipole antenna.

For practical, compact applications however, a planar antenna is preferred. A planar version of the spherical dipole antenna may be found in the 'two penny dipole antenna'.

\subsection{Two penny dipole antenna}

A circular planar dipole antenna may be constructed using two U.S. one cent pieces (pennies) and a semi-rigid coaxial piece of transmission line, [2,3], see figure 2 .
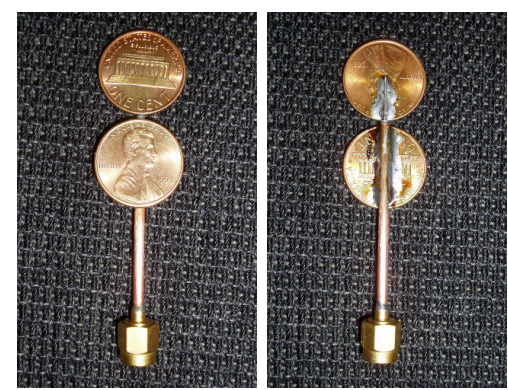

Figure 2: Two penny dipole antenna. Front and back. 
The measured return loss as function of frequency of this antenna is shown in figure 3 .

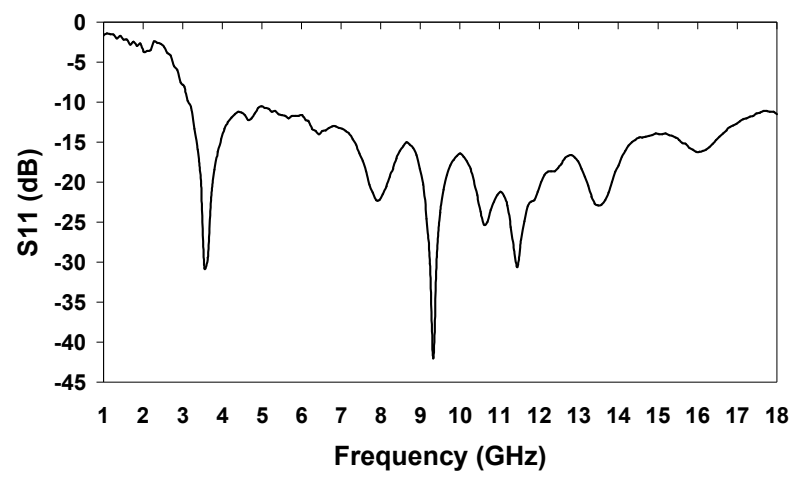

Figure 3: Measured return loss vs. frequency for the antenna shown in figure 2.

A good match $\left(S_{11}<-10 \mathrm{~dB}\right)$ may be observed for the UWB frequency band $(3.1 \mathrm{GHz}$ to $10.6 \mathrm{GHz})$ and beyond. The measurements show a first resonance just 'above' $3 \mathrm{GHz}$. Considering the size of the antenna - the diameter of a penny is $19 \mathrm{~mm}$ - this resonance may be attributed to the dipole. For higher frequencies, the good impedance match is due to the fact that the dipole with circular elements has transitioned to a dual-notch horn antenna, [2], see figure 4.
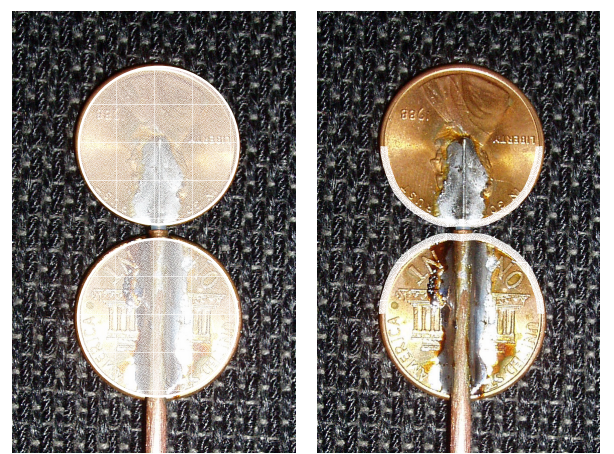

Figure 4: Two penny UWB antenna. Left. Dipole characteristic around $3 \mathrm{GHz}$. Right. Dual-notch horn characteristic for higher frequencies.

From the two penny UWB antenna it should be a relative small step towards the design of a compact PCB UWB antenna.

\section{PCB UWB antenna design}

A PCB microstrip version of the two-penny antenna has been realised. The upper circular dipole arm has been realised on the upper PCB plane and is connected to a microstrip transmission line, see figure 5. The lower circular dipole arm has been integrated with the microstrip ground plane, thus forming a pseudo-monopole, [5].

The lower circular arm has been integrated into a rectangular ground plane. This does not need to disturb the dual-notch horn antenna behaviour seriously, as long as the reflection level at the discontinuity formed by circle and rectangle is low. This reflection condition may be controlled by parameter $\mathrm{H}_{2}$, see figure 5 .

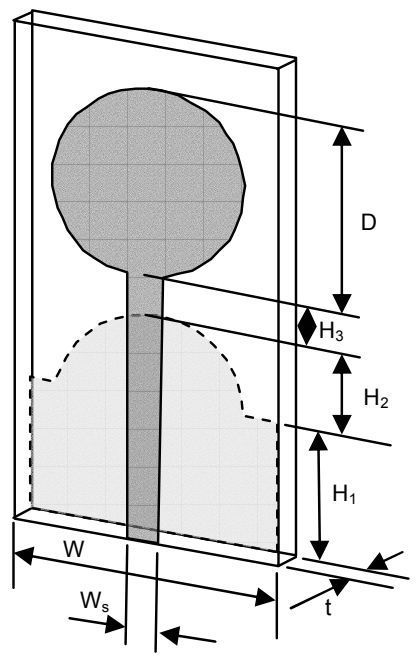

Figure 5: Microstrip UWB antenna.

The simulated return loss as function of frequency, employing CST Microwave Studio ${ }^{\circledR}$, is shown in figure 6. The used dimensions, see figure 5 , are stated in table 1 . The parameters $\varepsilon_{r}, \tan \delta$ and $t h$ are, respectively the relative permittivity of the PCB substrate, the loss tangent of the substrate and the thickness of the copper layers.

\begin{tabular}{|l|l|}
\hline Parameter & Value $(\mathrm{mm})$ \\
\hline$W$ & 22 \\
$W_{s}$ & 1.44 \\
$t$ & 1.6 \\
$t h$ & 0.07 \\
$H_{1}$ & 14.38 \\
$H_{2}$ & 4.62 \\
$H_{3}$ & 1.51 \\
$D$ & 19 \\
$\varepsilon_{r}$ & 4.28 \\
tan $\delta$ & 0.016 \\
\hline
\end{tabular}

Table 1: Dimensions microstrip UWB antenna.

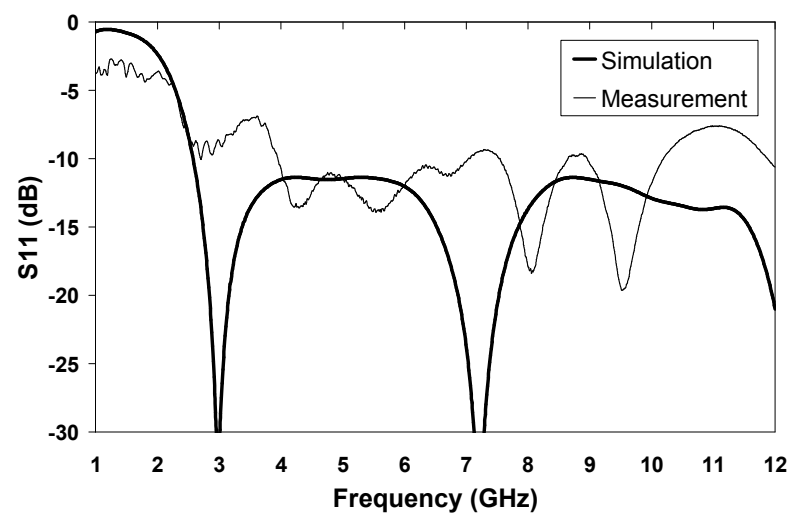

Figure 6: Simulated and measured return loss vs. frequency for a microstrip UWB antenna. 
The measurement results of the realised antenna, see figure 7 , are also shown in figure 6.
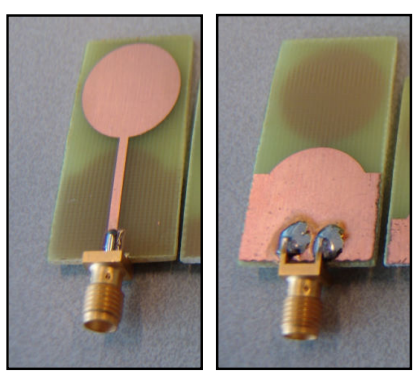

Figure 7: Realised microstrip UWB antenna. Left. Top. Right. Bottom.

During the measurements it was observed that cable currents highly influenced the measurement results. This is the main reason for the differences between simulations and measurements. Nevertheless, it is believed that with proper actions for suppressing these currents, [5,6], a close agreement may be reached. One has to bear in mind though that the antenna is intended for being applied in an integrated on-PCB solution.

\subsection{Feed line}

The disadvantage of the centre-fed dipole is that a transmission line must be brought to the gap between the dipole arms. Since the transmission line will be positioned inside the reactive near-field of the antenna, it will be vulnerable to undesired sheath coupling. The antenna radiation pattern may be distorted due to this coupling, [2]. In [2], a solution to this possible problem is shown including a strip transmission line feed and a tapered balun. In [4], a 'hidden' strip line feed is used. The strip line is positioned halfway between the two layers of the bottom dipole-arm. Both dipole arms consist of two metal layers on opposite sides of the PCB substrate, electrically connected through metallised vias located on the rims of the circular arms. This latter antenna will be used as benchmark (even though the circle diameter is $19.5 \mathrm{~mm}$, [4], where the diameter of 'our' circle is $19 \mathrm{~mm}$ ).

Our pseudo-monopole UWB antenna with non-hidden microstrip feed, however, does not seem to be prone to the above mentioned negative effects. This is demonstrated in figure 8 that shows the three-dimensional radiation patterns for $3 \mathrm{GHz}$ and $6 \mathrm{GHz}$. The gain value is indicated in the picture at the right. The antenna-PCB is positioned parallel to and in the $x y$-plane shown in figure 8 .

For the 'strip line dipole antenna' discussed in [4] as well as for our 'microstrip pseudo-monopole antenna' the radiation patterns for frequencies above $6 \mathrm{GHz}$ start to deviate from the half-wave dipole patterns shown in figure 8 . The reason for this deviation will be discussed later on. In table 2 the azimuth gain and maximum azimuth gain function variation for both antennas are shown for a number of discrete frequencies. The table demonstrates, together with figure 6 , that both antennas are comparable in behaviour.

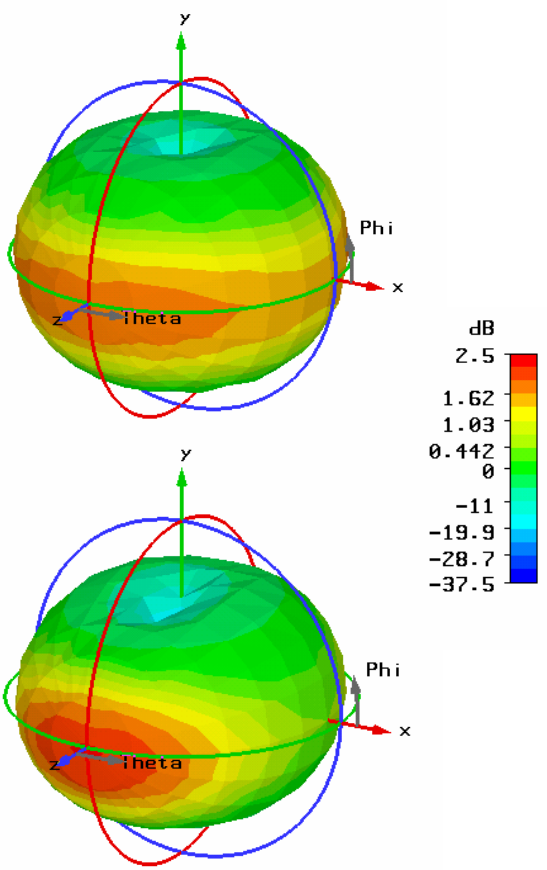

Figure 8: Three-dimensional radiation patterns of pseudomonopole printed UWB antenna. Top. $3 \mathrm{GHz}$. Bottom. $6 \mathrm{GHz}$.

\begin{tabular}{|l|l|l|l|l|}
\hline $\begin{array}{l}\text { Freq. } \\
(\mathrm{GHz})\end{array}$ & $\begin{array}{c}\text { Azimuth } \\
\text { gain }(\mathrm{dBi}) \\
\text { Stripline }\end{array}$ & $\begin{array}{c}\text { Azimuth } \\
\text { gain }(\mathrm{dBi}) \\
\text { Microstrip }\end{array}$ & $\begin{array}{c}\text { Max.var. } \\
(\text { dBi }) \\
\text { Stripline }\end{array}$ & $\begin{array}{c}\text { Max.var. } \\
(\text { dBi }) \\
\text { Microstrip }\end{array}$ \\
\hline 3 & 2.19 & 2.46 & 0.39 & 0.84 \\
4 & 2.63 & 3.02 & 0.79 & 1.69 \\
5 & 3.09 & 3.63 & 1.84 & 2.54 \\
6 & 3.82 & 4.02 & 3.26 & 3.35 \\
7 & 3.65 & 4.24 & 4.42 & 5.22 \\
8 & 2.46 & 2.16 & 5.10 & 8.16 \\
9 & - & -3.19 & - & 9.41 \\
10 & - & 0.11 & - & 15.91 \\
\hline
\end{tabular}

Table 2: Azimuth gain and maximum azimuth gain function variation as function of frequency for 'strip line dipole' and for 'microstrip pseudo-monopole'.

The gain as function of azimuth angle for the pseudomonopole antenna is shown in figure 9 for frequencies from $3 \mathrm{GHz}$ to $6 \mathrm{GHz}$. In figure 10 the gain as function of azimuth angle is shown for frequencies from $7 \mathrm{GHz}$ to $10 \mathrm{GHz}$.

Figure 10 clearly shows how for frequencies in excess of $6 \mathrm{GHz}$, the azimuth pattern seriously deviates from that of a half wave dipole antenna. Since the antenna behaves around $3 \mathrm{GHz}$ as a half wave dipole antenna, above $6 \mathrm{GHz}$ the length of the antenna gets larger than a wavelength and elevational lobes will evolve with increasing frequency. This is 
demonstrated in figure 11 , showing the three-dimensional radiation patterns at $7 \mathrm{GHz}$ and at $10 \mathrm{GHz}$.

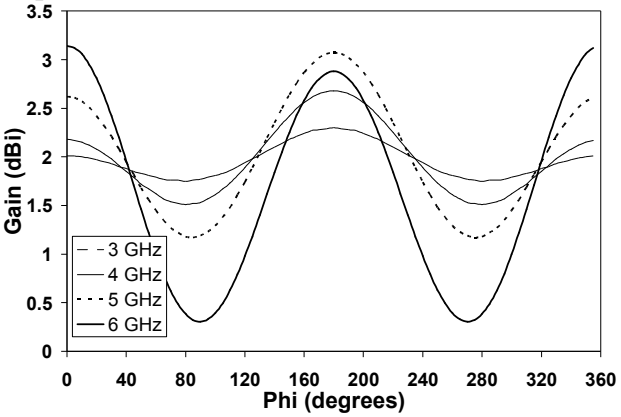

Figure 9: Gain vs. azimuth angle for pseudo-monopole printed UWB antenna for $3 \mathrm{GHz}, 4 \mathrm{GHz}, 5 \mathrm{GHz}$ and $6 \mathrm{GHz}$.

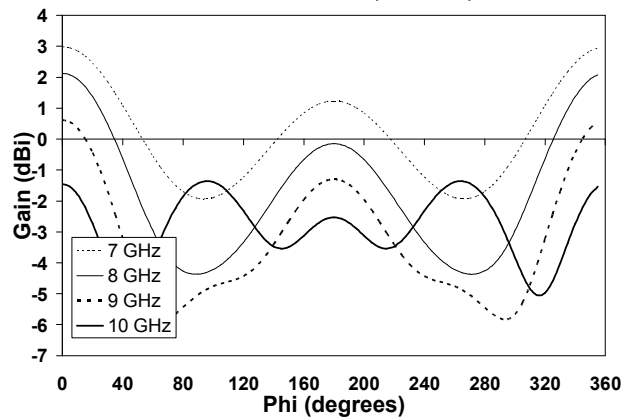

Figure 10: Gain vs. azimuth angle for pseudo-monopole printed UWB antenna for $7 \mathrm{GHz}, 8 \mathrm{GHz}, 9 \mathrm{GHz}$ and $10 \mathrm{GHz}$.

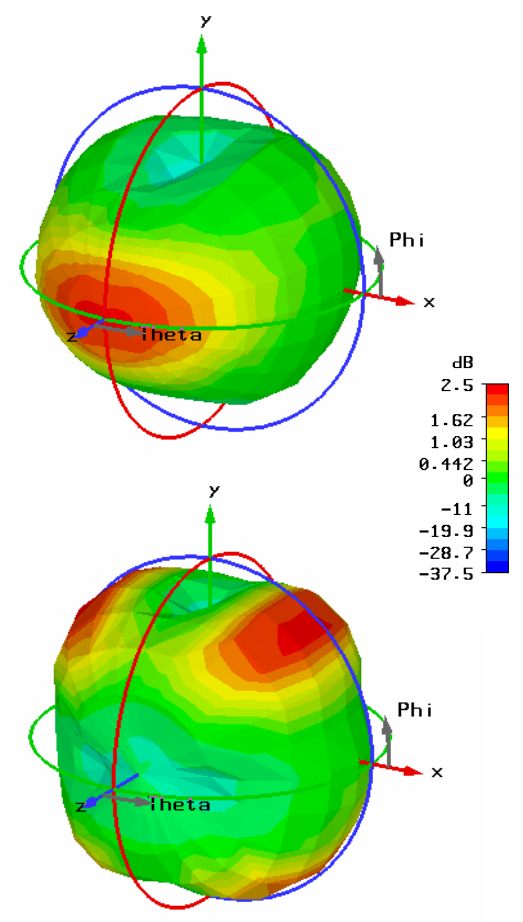

Figure 11: Three-dimensional radiation patterns of pseudomonopole printed UWB antenna. Top. 7GHz. Bottom. $10 \mathrm{GHz}$
A way of creating a more dipole like pattern over the whole USB frequency band is accomplished by shortening the antenna.

\subsection{Antenna shortening}

When we shorten the antenna, the generation of elevational lobes will start at a higher frequency. The first resonance, however, will also occur at a higher frequency. Looking at the return loss vs. frequency (simulation) results, figure 6 , we see that we still have some margin if we require a return loss of less than $-10 \mathrm{~dB}$ over the frequency band of $3.1 \mathrm{GHz}$ to $10.6 \mathrm{GHz}$. Figure 12 shows the simulated and measured return loss vs. frequency results for a shortened pseudo monopole antenna. This antenna is created by changing $H_{1}=14.38 \mathrm{~mm}$ into $H_{l}=6.38 \mathrm{~mm}$.

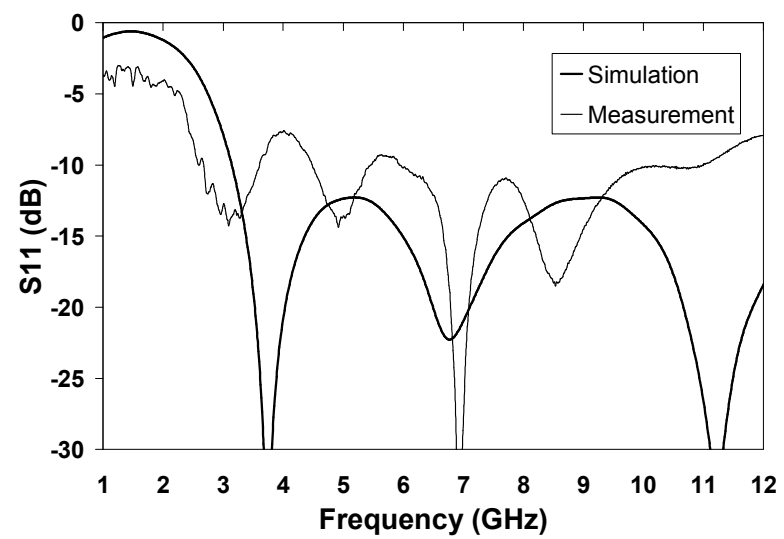

Figure 12: Simulated and measured return loss vs. frequency for shortened microstrip UWB antenna.

The measurements again suffered from cable current effects. Comparing the simulation results with those shown in figure 6 , however, show that it is possible to shorten the antenna without compromising the return loss characteristics. One has to be careful though for the return loss around $3 \mathrm{GHz}$. In table 3 we compare the azimuth gain characteristics of the shortened pseudo-monopole antenna with those of the strip line dipole antenna, [4]

\begin{tabular}{|l|l|l|l|l|}
\hline $\begin{array}{l}\text { Freq. } \\
(\mathrm{GHz})\end{array}$ & $\begin{array}{c}\text { Azimuth } \\
\text { gain }(\mathrm{dBi}) \\
\text { Stripline }\end{array}$ & $\begin{array}{c}\text { Azimuth } \\
\text { gain }(\mathrm{dBi}) \\
\text { Short } \\
\text { Microstrip }\end{array}$ & $\begin{array}{l}\text { Max.var } \\
\text { (dBi) } \\
\text { Stripline }\end{array}$ & $\begin{array}{c}\text { Max.var. } \\
(\text { dBi) } \\
\text { Short } \\
\text { Microstrip }\end{array}$ \\
\hline 3 & 2.19 & 2.30 & 0.39 & 0.55 \\
4 & 2.63 & 2.68 & 0.79 & 1.17 \\
5 & 3.09 & 3.07 & 1.84 & 1.90 \\
6 & 3.82 & 3.14 & 3.26 & 2.84 \\
7 & 3.65 & 2.99 & 4.42 & 5.58 \\
8 & 2.46 & 2.13 & 5.10 & 6.50 \\
9 & - & 0.62 & - & 6.45 \\
10 & - & 1.36 & - & 3.68 \\
\hline
\end{tabular}

Table 3: Azimuth gain and maximum azimuth gain function variation as function of frequency for 'strip line dipole' and for the shortened 'microstrip pseudo-monopole'. 
The table shows that the azimuth gain behaviour has improved with respect to the original microstrip pseudomonopole antenna. This is again demonstrated in figures 13 that shows the maximum gain and maximum gain variation as function of frequency for the 'long' and 'short' pseudomonopole antenna.

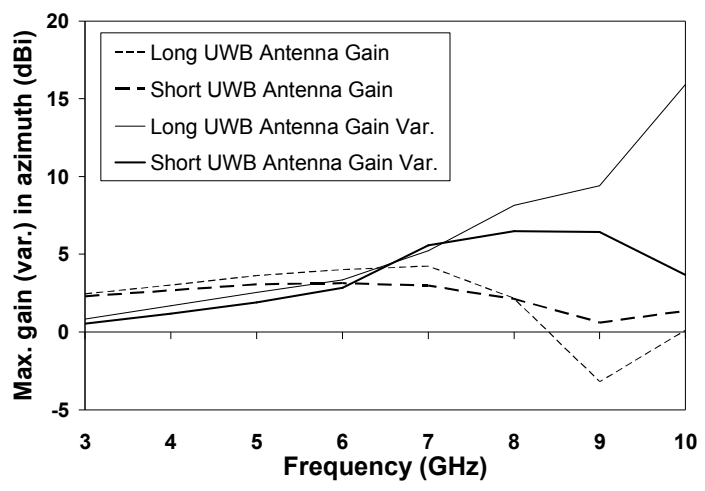

Figure 13: Simulated maximum gain and maximum gain variation in azimuth vs. frequency for original (long) and shortened (short) microstrip UWB antenna.

The figure shows that the shortened antenna exhibits a more constant gain over the frequency band. A comparison of table 3 with table 2 further learns that the shortened microstrip UWB antenna shows a behaviour more similar to that of the strip line UWB antenna. The dimensions however are smaller and the antenna is easier to produce and does not have a critical, [7], feeding and transition region.

With this shortened microstrip pseudo-monopole UWB antenna as basis, we will take measures to suppress signals in the $5 \mathrm{GHz}$ to $6 \mathrm{GHz}$ frequency band.

\section{Band-stop filter}

To create a frequency band-notch function we may either change the current flow on the metal parts of the antenna, or we may insert a filter before or in the feed line of the antenna.

\subsection{Slot in radiator}

To influence the current flow, we will introduce a slot into the upper arm of our antenna. Since, for higher frequencies, the current will concentrate at the rims of the two circular arms, the slot has to be positioned in the neighbourhood of the circle rim. For the ease of drawing we have chosen for a Ushaped slot as shown in figure 14. The slot dimensions, after optimisation, are as stated in table 4.

\begin{tabular}{|l|l|}
\hline Parameter & Value $(\mathrm{mm})$ \\
\hline$W_{U 1}$ & 7.2 \\
$W_{U 2}$ & 1.2 \\
$W_{L}$ & 1.2 \\
$L_{U 1}$ & 15.1 \\
$L_{U 2}$ & 5.9 \\
\hline
\end{tabular}

Table 1: Slot dimensions microstrip UWB antenna.

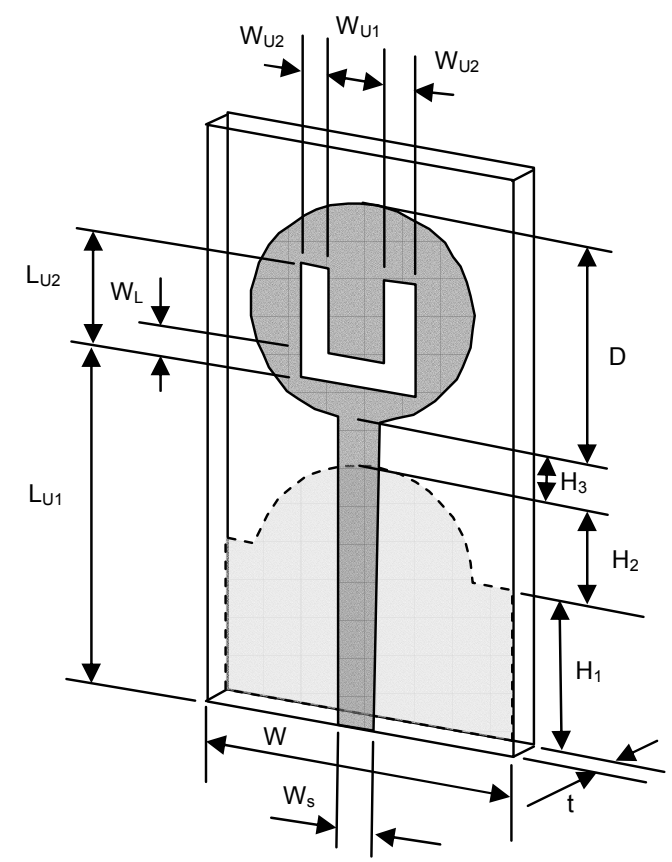

Figure 14: Microstrip UWB antenna with U-shaped slot for frequency band-notch function. Dimensions.

The simulated and measured return loss as function of frequency is shown in figure 15 .

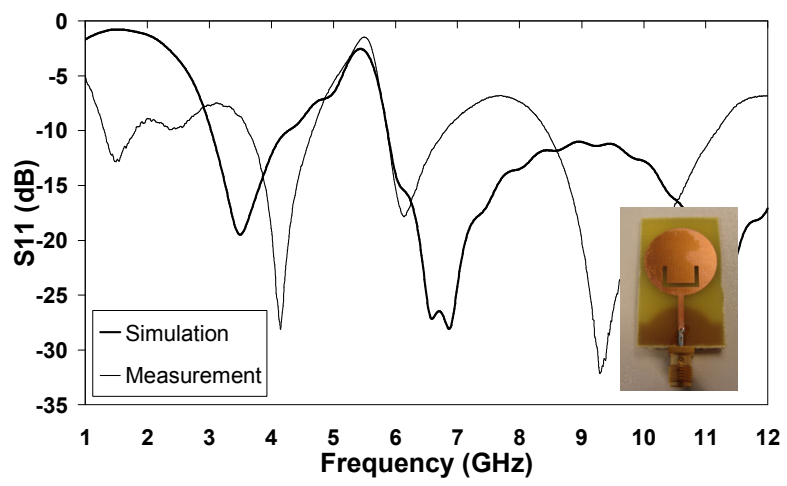

Figure 15: Simulated and measured return loss vs. frequency for short microstrip UWB antenna with U-shaped slot.

Although the measurements are still hindered by cable current effects, we clearly see how the U-shaped slot ads the desired frequency band-noth functionality. The prototype antenna is shown as inset in the figure.

\subsection{Spurline filter in microstrip}

A microstrip spurline filter, [8], acts as a band stop filter. A nice feature of a spurline filter is that the physical structure is completely contained within the boundaries of the microstrip transmission line, see figure 16 .

The spurline filter reflection and transmission may be relatively easy calculated using the closed formulas for the elements of the ABCD matrix given in [8]. In these equations 
use may be made of the static even and odd mode effective permittivities and characteristic impedances for parallel coupled microstrip lines of $[9,10]$.

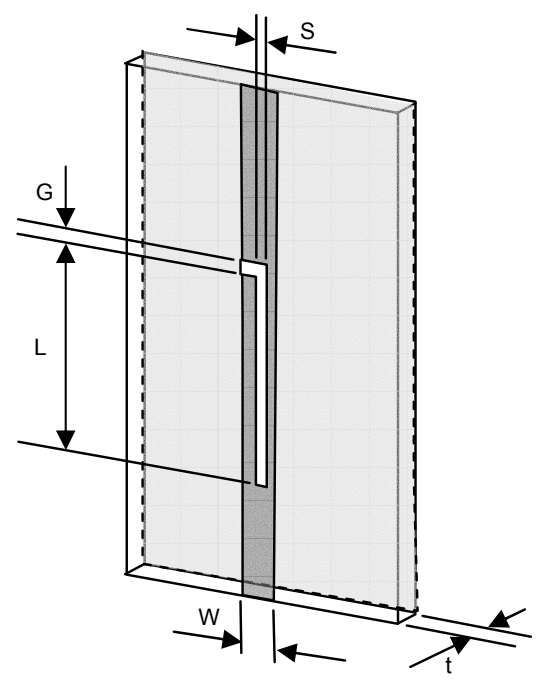

Figure 16: Microstrip spurline filter.

As an example, in figure 17 , the transmission coefficient vs. frequency for a spurline filter with $W=3.3 \mathrm{~mm}, S=G=0.3 \mathrm{~mm}$, $L=15 \mathrm{~mm}, t=1.6 \mathrm{~mm}, \varepsilon_{r}=4.28, \operatorname{th}=0.07 \mathrm{~mm}$ and $\tan \delta=0.016$ as calculated with the closed form equations is compared with Method of Moments simulation results. The agreement is good.

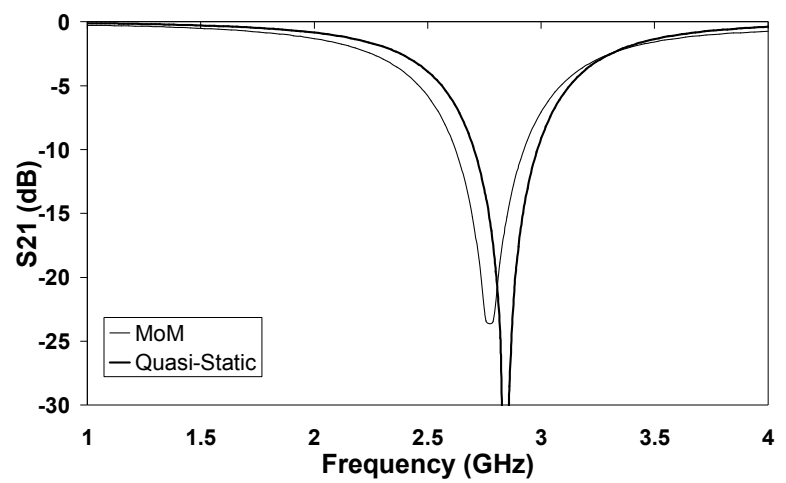

Figure 17: Transmission coefficient vs. frequency as calculated for a microstrip spurline filter.

Since te spurline filter is completely contained within the microstrip, we may bring the filter into the feeding structure of the antenna itself. As first - non-optimised - simulation results reveal, the coupling with the radiating structure needs to be taken into account, see figure 18 .

Although additional design work is needed, the figure reveals that it should be possible to design a compact UWB antenna with integrated spurline filter.

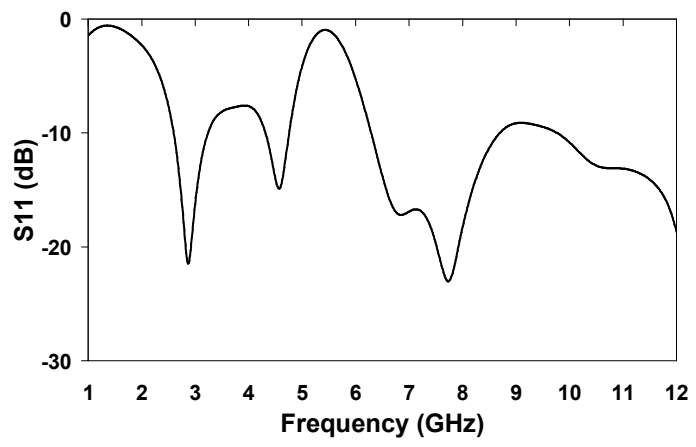

Figure 18: Simulated return loss vs. frequency for original (non-shortened) pseudo-monopole UWB antenna with integrated spurline filter.

\section{Conclusions}

Low-cost, compact, circular planar dipole UWB antennas have been designed. The use of microstrip in stead of stripline technology hardly degrades the radiation characteristics, but simplifies the antenna architecture considerably. By inserting a slot in the upper arm of the dipole or inserting a spurline filter in the microstrip part of the antenna, a stop band from $5 \mathrm{GHz}$ to $6 \mathrm{GHz}$ may be realised.

\section{Acknowledgements}

The author would like to thank Ad Reniers for his assistance in simulations and measurements.

\section{References}

[1] C. A. Balanis, "Antenna Theory, Analysis and Design, $2^{\text {nd }}$ edition", John Wiley \& Sons, New York, (1996).

[2 H. Schantz, "The Art and Science of Ultrawideband Antennas", Artech House, Boiston, USA, (2005).

[3] www.timederivative.com/PUBs-2cent-antenna.pdf

[4] E. Gueguen, F. Thudor and P. Chambelin, "A Low Cost UWB Printed Dipole Antenna with High Performances", IEEE Int. Conf. UWB, pp. 89-92, (2005)

[5] T. W. Hertel, "Cable-Current Effects of Miniature UWB Antennas", IEEE AP-S Int. Symp., pp. 524-527, (2005).

[6] D. -H. Kwon and Y. Kim, "Suppression of Cable Leakage Current for Edge-Fed Printed Dipole UWB Antennas Using Leakage-Blocking Slots", IEEE Ant. Wireless Prop. Letters, Vol. 5, pp. 183-186, (2006).

[7] www.cst.com/Content/Applications/Article/Ultra-WideBand+Printed+Circular+Dipole+Antenne

[8] N. Nguyen, C. Hsieh and D. W. Ball, "Millimeter Wave Printed Circuit Spurline Filters", MTT-S Int. Microwave Symp. Digest, pp. 98-100, (1983).

[9] M. Kirschning and R. H. Jansen, "Accurate Wide-Range Design Equations for the Frequency-Dependent Characteristic of Parallel Coupled Microstrip Lines" IEEE Trans. Microw. Theory Techn., Vol. 32, No. 1, pp. 83-90, (1984).

[10] M. Kirschning and R. H. Jansen, Corrections to [9]. 